\title{
Gene Loss, Protein Sequence Divergence, Gene Dispensability, Expression Level, and Interactivity Are Correlated in Eukaryotic Evolution
}

\author{
Dmitri M. Krylov, Yuri I. Wolf, Igor B. Rogozin, and Eugene V. Koonin ${ }^{1}$ \\ National Center for Biotechnology Information, National Library of Medicine, National Institutes of Health, \\ Bethesda, Maryland 20894, USA
}

\begin{abstract}
Lineage-specific gene loss, to a large extent, accounts for the differences in gene repertoires between genomes, particularly among eukaryotes. We derived a parsimonious scenario of gene losses for eukaryotic orthologous groups (KOGs) from seven complete eukaryotic genomes. The scenario involves substantial gene loss in fungi, nematodes, and insects. Based on this evolutionary scenario and estimates of the divergence times between major eukaryotic phyla, we introduce a numerical measure, the propensity for gene loss (PGL). We explore the connection among the propensity of a gene to be lost in evolution (PGL value), protein sequence divergence, the effect of gene knockout on fitness, the number of protein-protein interactions, and expression level for the genes in KOGs. Significant correlations between PGL and each of these variables were detected. Genes that have a lower propensity to be lost in eukaryotic evolution accumulate fewer substitutions in their protein sequences and tend to be essential for the organism viability, tend to be highly expressed, and have many interaction partners. The dependence between PGL and gene dispensability and interactivity is much stronger than that for sequence evolution rate. Thus, propensity of a gene to be lost during evolution seems to be a direct reflection of its biological importance.
\end{abstract}

Lineage-specific gene loss is one of the major evolutionary processes that have been brought to light by comparative analyses of gene sets from completely sequenced genomes (Aravind et al. 2000; Moran 2002). The extent of gene loss can be dramatic, and it can occur relatively rapidly under a strong selective pressure. For example, the endosymbiotic bacterium Buchnera aphidicola has 580 genes compared with the $\sim 4300$ genes in the genome of the closely related $\gamma$-proteobacterium Escherichia coli. Apparently, Buchnera has lost $\sim 86 \%$ of the genes during its adaptation to the endosymbiotic life style, to which this bacterium converted 200 to 250 million years ago (Baumann et al. 1995). Similarly, the genome of a eukaryotic intracellular parasite, the microsporidian Encephalitozoon cuniculi, contains $\sim 2000$ genes, compared with 5500 to 6000 genes in the genomes of yeasts, which themselves probably have undergone considerable gene loss (Katinka et al. 2001). Although genomes of parasites expose the most striking cases of massive gene loss, recent reconstructions of parsimonious scenarios of evolution for prokaryotes indicated that substantial gene loss has occurred in all phylogenetic lineages (Snel et al. 2002; Mirkin et al. 2003). In prokaryotes, gene loss is one of the two major evolutionary processes, along with horizontal gene transfer (HGT), that contribute to the intensive "gene flux" that seems to have shaped the genomes of these organisms. In eukaryotes, particularly in complex multicellular organisms, the evolutionary significance of lineage-specific gene loss might be even greater because HGT between these organisms does not appear to be widespread. The likelihood that a gene is lost during evolution, which is reflected in the pattern of presence-absence of the gene in the analyzed genomes (hereinafter, phyletic pattern), appears to be an important measure of evolutionary conservation.

Sequence divergence is a measure of the evolutionary conservation of a gene that is fundamentally different from gene loss

\footnotetext{
'Corresponding author.

E-MAIL koonin@ncbi.nlm.nih.gov; FAX (301) 480-9241.

Article and publication are at http://www.genome.org/cgi/doi/10.1101/ gr.1589103.
}

propensity. Although gene loss is the result of a complete deletion or oblation of a gene, sequence divergence occurs through point mutations, as well as small deletions and insertions, and generally does not lead to elimination of the gene. Hence, these two variables, gene loss propensity and sequence divergence (or its correlate, the evolutionary rate), seem to be complementary measures of the conservation of a gene during evolution. Sets of orthologous proteins show a broad distribution of evolutionary rates (Grishin et al. 2000; Bromham and Penn 2003; Hedges and Kumar 2003). For example, protein sequences of ubiquitins or histones in eukaryotes typically are 90\%-98\% identical, whereas dihydroorotases (essential enzymes of pyrimidine metabolism) are only $20 \%$ to $30 \%$ identical.

The evolutionary rate of a gene, that is, the estimated number of substitutions per position between orthologous sequences, has long been assumed to depend on the importance of the gene in question for the fitness of the organism. The "knockout rate" hypothesis predicts that the greater the effect of a gene knockout on fitness, the slower the evolutionary rate. In particular, essential genes (those for which knockout is lethal) are expected to evolve significantly slower than are nonessential ones (Wilson et al. 1977). The availability of multiple genome sequences and genome-wide data on the phenotypes of gene knockouts for model organisms, such as yeast Saccharomyces cerevisiae (Giaever et al. 2002) and the nematode Caenorhabditis elegans (Kamath et al. 2003), enabled direct testing of these predictions. More generally, comparative analyses aimed at the identification of characteristics of genes that determine or at least strongly correlate with the evolutionary rate have become feasible. The results of the tests of the knockout rate hypothesis have been somewhat contradictory, but the studies with larger samples of genes indeed revealed a positive correlation between the evolutionary rate and the effect of a gene knockout on the fitness of the organism (Hirsh and Fraser 2001). However, it appeared rather unexpectedly that the effect was relatively minor, although statistically significant thanks to the large amounts of data analyzed; that is, only a small part of the variability of the evolutionary rate could be explained by differences in gene dispensability. 
We sought to investigate the connection between the two distinct measures of the evolutionary conservation of a gene: (1) the newly introduced propensity for gene loss (PGL) and the rate of sequence evolution and (2) the major variables that determine the functional importance of a gene, namely, the effect of gene knockout on fitness, interactivity, and expression level. For this analysis, we used the recently developed collection of clusters of eukaryotic orthologous groups (KOGs) of proteins from seven (nearly) completely sequenced eukaryotic genomes (Tatusov et al. 2003), which allowed us to construct a parsimonious scenario of gene losses along the branches of the eukaryotic phylogenetic tree. We introduce here a numerical measure for gene loss, PGL, and show a statistically significant positive correlation between PGL and evolutionary rate of a KOG. Furthermore, both PGL and sequence divergence strongly and negatively correlate with the fitness effect of knockout, interactivity, and expression level of the respective gene. The protein sequences of genes that are rarely lost during evolution change relatively slowly; these genes tend to be essential for the survival of an organism and are highly expressed.

\section{RESULTS}

\section{The Data Set of Conserved KOGs and Distribution} of Gene Losses Over the Eukaryotic Phylogenetic Tree The KOG database contains 5873 KOGs represented in two to seven eukaryotic genomes: the plant Arabidopsis thaliana; animals C. elegans, Drosophila melanogaster, and Homo sapiens; fungi $S$. cerevisiae and Schizosaccaromyces pombe; and the microsporidian E. cuniculi (Tatusov et al. 2003; http://www.ncbi.nlm.nih. gov/COG/new/shokog.cgi). According to the phylogeny of the eukaryotic crown group that is currently considered most likely (Hedges 2002), plants branched off first, followed by the divergence of the fungi-microsporidian and metazoan (animal) clades (Fig. 1). For the purposes of the present analysis, we chose a subset of KOGs that are represented in at least three species and could be traced back to the last common ancestor of plants, animals, and fungi. If the amount of HGT between complex eukaryotes is considered to be negligible, reconstruction of the ancestral gene set becomes straightforward: All 3140 KOGs shared by Arabidopsis and any two of the other species should be considered ancestral (KOGs consisting of only two species were not analyzed).

Given a tree topology, the most parsimonious evolutionary scenario resulting in the observed distribution of the phyletic patterns of KOGs can be reconstructed by using the evolutionary parsimony principle. For the purpose of this reconstruction, the phyletic pattern of each KOG was treated as a string of binary characters (one, the presence of the given species; zero, its absence in the given KOG). Given the implausibility of HGT between eukaryotes, the Dollo parsimony principle, under which gene loss is treated as irreversible (a gene can be lost independently in several evolutionary lineages but cannot be regained), was adopted (Farris 1977).

In the resulting parsimonious scenario, each branch was associated with the number of gene losses such that the sum total of losses was minimal, with the exception of the plant branch and the branch leading to the common ancestor of fungi and animals: Gene losses could not be assigned to these branches with the current set of genomes (Fig. 1). The evolutionary scenario includes a massive gene loss in the fungal clade, with additional loss in the microsporidian, and subsequent substantial gene loss in each of the animal lineages, particularly in the nematodes and arthropods (Fig. 1).
A


Figure 1 The phylogeny of eukaryotes and PGL calculations. (A) Estimated divergence times in millions of years ago (MYA) are shown for all internal nodes of the tree; the estimates are from Hedges et al. (2001). The number of lost genes according to the reconstructed parsimonious scenario is shown next to each branch. $(B, C)$ Examples of PGL calculation. The presence and absence of a gene in each of the extant species is indicated by " + " and " - ", respectively. Red branches are those that retained the gene; blue branches are those to which a loss was mapped. (B) The loss of gene in the branch leading to the common ancestor of yeasts and microsporidian is shown by a blue dot because this branch formally has zero length.

\section{Propensity for Gene Loss}

The simplest numerical measure for gene loss in a group of orthologs is the fraction of lineages in which a given gene has been lost. However, the one/zero scoring scheme for gene loss and preservation in different lineages does not reflect the time during which a particular gene was lost or preserved. This time can be different for different lineages, which renders the binary measure inaccurate. In our reconstruction of the parsimonious evolutionary scenario, we mapped gene losses onto the widely accepted phylogenetic tree for the analyzed lineages. The PGL for each gene (KOG) was then calculated by taking into account the tree topology and the available time estimates for each divergence point (Hedges et al. 2001; Hedges 2002; Hedges and Kumar 2003). The logic behind this calculation was as follows. Each branch of the phylogenetic tree was treated as an independent trial during which the given gene was either preserved or lost. The longer the time during which a gene could have been lost, but was not, compared with the total time available, the lower the propensity of this gene to be lost (Fig. 1; for details, see Methods).

A PGL value of zero corresponds to KOGs that are represented in all seven species. A PGL value of one, in theory, would be assigned to a gene present in the last common ancestor of the analyzed species but lost in all lineages. Such genes, for obvious reasons, cannot be detected, and in practice, PGL values can

\section{Genome Research www.genome.org}


range from zero to some maximum value less than one. In the data set analyzed here, the PGL values varied from zero to 0.49 , the upper limit of PGL being a function of the number of lineages included and the times since their divergence. Genes with PGL value that was estimated as zero using the current data set of seven species (i.e., that were not lost in any of these seven species) might, in reality, have some propensity to be lost in other species. Nevertheless, the PGL values remain meaningful and internally consistent for this data set inasmuch as they are used to estimate the relative propensity for gene loss among all analyzed genes over the time elapsed since the last common ancestor of the compared species. The highest PGL value obtained here, 0.49 , is the maximum only for the genes and species considered in this analysis; as additional genomes are included, greater PGL values will result.

\section{The Dependence Between Gene Loss and Sequence Evolution Rate}

The tendency of a gene to be lost and sequence evolution rate are two variables that characterize the evolutionary conservation of the gene. A priori, these variables could be considered independent. For example, a protein potentially could evolve relatively fast due to relaxed functional constraints but have a low propensity for loss linked to an essential function. For the purposes of the present analysis, we used the mean evolutionary distance between the KOG member from Arabidopsis (the outgroup with respect to the other analyzed species; Fig. 1) and the rest of the KOG members as the measure of the sequence evolution rate characteristic of the KOG (gene) as a whole. When the PGL values for the analyzed sample of 3140 KOGs were plotted against the evolutionary rates (determined with several methods, see Methods), clear positive correlation was observed (Table 1). The correlation coefficient $(\mathrm{R})$ ranged from $0.3-0.4$, depending on the distance measure used, whereas all correlations were statistically highly significant $\left(p<<10^{-6}\right)$. Thus, the assumption of independence of the two variables could be rejected with a high degree of confidence. There is a definite connection between the two facets of evolutionary conservation: The more often a gene is lost, the more substitutions it typically accumulates. However, it is equally notable that the interdependence of the two values is not overwhelmingly strong as only $10 \%-15 \%$ of the variation in the sequence evolution rate can be explained by variation in PGL (and vice versa).

\section{Viability of Knockouts of Yeast Genes With Different Propensities for Loss}

Intuitively, it appears that the propensity of a gene to be lost should strongly correlate with the effect of gene knockouts on the viability of the organism. Indeed, one would surmise that if a gene is never lost during a long span of evolution, this is because its function is essential for survival. The PGL values for those KOGs that are represented in S. cerevisiae were superimposed over the available data on the effect of gene knockout on yeast viability (Giaever et al. 2002). More than half of the genes with PGL equal to zero, that is, those that have not been lost in any of the seven lineages considered here, are essential; that is, the respective knockouts are lethal (Fig. 2). The fraction of essential genes was dramatically lower in all other PGL classes $\left(P<<10^{-6}\right.$ by the $\chi^{2}$ criterion). Thus, genes with the lowest propensity for loss during evolution seem to be involved in indis-

pensable functions to a much greater extent than are those genes that have been lost in some lineages. Although one might expect that the fraction of essential genes among those with $\mathrm{PGL}=0$ could be somewhat lower in more complex organisms due to functional redundancy among paralogs, the conservation pattern of a gene expressed numerically through PGL still could be a reasonable predictor of essential gene functions.

In contrast to the strong connection between the PGL and (in)dispensability of a gene, and in agreement with the previous report (Hirsh and Fraser 2001), we found no appreciable correlation between the sequence evolution rate and dispensability. Among the genes with $\mathrm{PGL}=0$, the sequence evolution rate was slightly lower for essential genes, but the difference in rates between essential and nonessential genes was statistically significant $(p<0.05)$ for only one method of evolutionary rate calculation, the PAM distances (Table 2). Thus, although PGL positively, and strongly, correlates with both sequence evolution rate and dispensability, the latter two variables are not significantly correlated; that is, they appear to be (nearly) independently linked to PGL.

\section{Propensity for Gene Loss, Substitution Rates, and Expression Levels}

A highly significant negative correlation between the evolutionary rate of yeast genes has been reported: Highly expressed genes appear to evolve slowly (Pal et al. 2001). We examined the correlation between the gene expression levels in various organisms, PGL, and the sequence evolution rate. A significant negative correlation was detected between the expression level and both measures of evolutionary conservation; that is, highly expressed genes tend to evolve more slowly and to be less prone to loss in various lineages than are genes expressed at lower levels. Although the correlation coefficient varied for different measures of evolutionary distance, it was consistently greater for sequence evolution rate than for PGL (Table 1).

\section{Number of Protein-Protein Interactions, PGL, and Substitution Rates}

Genes with products that are involved in numerous proteinprotein interactions tend to evolve more slowly than do those that have few interaction partners, although the magnitude of the difference varied in different studies and was not dramatic in any of them (Fraser et al. 2002; Jordan et al. 2003). We examined the correlation between PGL and sequence evolution rate, on the one hand, and the number of protein-protein interactions for the KOG members from yeast on the other hand. To this end, the data set collected in the General Repository for Interaction Datasets (GRID) database (Breitkreutz et al. 2003) was used as the

Table 1. Correlation (R) Between the Propensity for Gene Loss, Substitution Rates, Gene Expression Level, and the Number of Protein-Protein Interactions

\begin{tabular}{lccccc}
\hline & & \multicolumn{3}{c}{ Expression in } & \\
\cline { 3 - 5 } & PGL & Yeast & Worm & Human & Interactions \\
in yeast
\end{tabular}

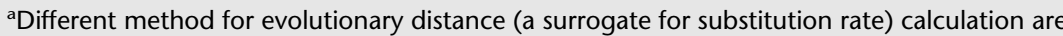
introduced in Methods.

${ }^{\mathrm{b}}$ A.t., Arabidopsis thaliana. 


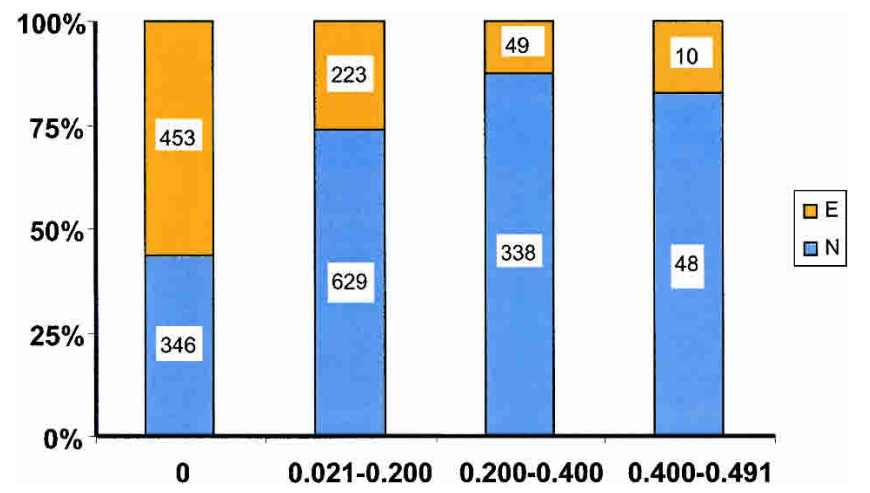

Figure 2 Distribution of essential and nonessential yeast genes among PGL classes. Yeast proteins were binned into four classes according to the $\mathrm{PGL}$ values for the corresponding KOGs. The number of essential (E) and nonessential $(\mathrm{N})$ genes in each class is indicated. If there were multiple yeast paralogs in a KOG, the KOG was counted as essential if at least one of the paralogs was essential.

source of protein-protein interaction data. We found a strong negative correlation between the number of protein-protein interactions per protein and PGL, and a weaker correlation with various measures of sequence evolution rate (Table 1). Both correlations were highly statistically significant $\left(P<10^{-6}\right)$. Furthermore, when the KOGs were binned according to their PGL values, the difference in the mean number of interactions of yeast proteins between the bins appeared dramatic (Fig. 3). Thus, proteins that have many interaction partners seem to be substantially less prone to loss during evolution than are those with fewer partners, and this connection is much stronger than that between the interactivity and sequence evolution rate. This is compatible with the observation that highly connected proteins in the yeast interaction network include a higher proportion of essential gene products than do proteins with fewer interactions (Jeong et al. 2001).

\section{DISCUSSION}

Sequence evolution rate is a traditional measure of the conservation during evolution of a gene. Early molecular evolutionary studies have unequivocally shown that different genes evolve at substantially different rates (Kimura 1983). However, only with the advent of genomics and other kinds of "omics", such as genome-wide analysis of gene expression and protein-protein interactions, has the opportunity presented itself to systematically explore the connections between the evolution rate and various other characteristics of genes (Wolfe and Li 2003). The results of these studies so far have been somewhat disappointing, in that a truly strong correlate of the evolution rate had not been identified. It has been shown that slow-evolving genes tend to be highly expressed (Pal et al. 2001) and encode longer proteins (Lipman et al. 2002) that tend to be involved in a somewhat greater number of protein-protein interactions than are fastevolving gene products (Fraser et al. 2002; Jordan et al. 2003). However, establishing the significance of each of these correlations required careful examination of statistical evidence. In other words, none of these correlations is particularly strong, and none can explain much of the variation in evolution rate, although they are statistically significant thanks to the massive amounts of genomics data. Notably, the results of direct tests of Wilson's knockout rate hypothesis are in the same category: Knockout of slow-evolving genes tends to have a greater effect on fitness than does knockout of fast-evolving genes, but the con- nection is relatively weak, to the point that some studies have failed to support its significance (Hurst and Smith 1999; Hirsh and Fraser 2001; Jordan et al. 2002; Pal et al. 2003).

These observations incite the iconoclastic idea that sequence evolution rate might not be the most biologically relevant measure of the evolutionary conservation of a gene. Here we explored an alternative, the propensity of a gene to be lost during evolution, a characteristic that obviously can be measured only through comparison of multiple complete gene sets. PGL is a much more intuitive correlate of the dispensability of a gene than is sequence evolution rate; indeed, if a gene is never lost during evolution, that is probably because it is essential for viability. However, the connection is not as trivial as it seems to be at first glance because it is based on a strong assumption, namely, the transfer of the information on the essentiality of a gene in one organism (e.g., yeast) to its ortholog in another, vastly different organism (e.g., worm). Actually, the conservation of essentiality is not guaranteed because a gene might be rendered nonessential by the evolution of redundancy, in the form of paralogs or unrelated but functionally analogous genes. This might be followed by the loss of a formerly essential gene, resulting in nonorthologous gene displacement (Koonin and Mushegian 1996).

Empirically, we observed a strong connection, but definitely not a one-to-one correspondence, between PGL and knockout viability, and a highly significant positive correlation between PGL and sequence evolution rate. In contrast, sequence evolution rate and viability are linked weakly at best. This suggests that PGL carries with it a strong biological signal, which is directly linked to the dispensability of a gene and less directly, even if indisputably, to the sequence evolution rate. By transitivity, it should be expected that the latter two variables are also correlated but that connection is nearly lost in the statistical noise. Thus, a gene shown to be essential in a particular organism has a strong tendency to be retained and, by implication, to be essential even in phylogenetically remote lineages; the protein sequences encoded by such genes also might tend to evolve slightly slower than do those of nonessential genes.

These conclusions are supported by the detected strong correlation between PGL and the interactivity of a protein: Hubs of the protein interaction network are lost during evolution much less readily than are proteins with few interaction partners, and this connection is much stronger than that between interactivity



Figure $3 \mathrm{PGL}$ and number of protein-protein interactions for yeast proteins. Yeast proteins were binned into four classes according to the PGL values for the corresponding KOGs. The average number of interactions was calculated for each class. For KOGs with multiple yeast paralogs, the sum of interactions for all paralogs was used, with the rationale that this is the natural integral measure of the interactivity of the proteins in the given KOG, under the assumption that all paralogs in a KOG have evolved via relatively recent, lineage-specific duplications.

\section{Genome Research \\ www.genome.org}


Table 2. Viability of Knockouts in Yeast, PGL, and Sequence Evolution Rate

\begin{tabular}{|c|c|c|c|c|c|c|c|c|c|c|}
\hline \multirow[b]{2}{*}{ PGL/lethality } & \multicolumn{2}{|c|}{ P-distance $^{a}$} & \multicolumn{2}{|c|}{ JTT } & \multicolumn{2}{|c|}{ JTT, three-kingdom average } & \multicolumn{2}{|c|}{ JTT, $\gamma$-corrected } & \multicolumn{2}{|c|}{ PAM } \\
\hline & - & + & - & + & - & + & - & + & - & + \\
\hline 0 & 0.559 & 0.558 & 1.198 & 1.221 & 1.005 & 1.026 & 1.975 & 2.048 & 0.903 & 0.960 \\
\hline $0.02-0.2$ & 0.635 & 0.625 & 1.505 & 1.457 & 1.361 & 1.324 & 2.662 & 2.535 & 1.126 & 1.099 \\
\hline $0.2-0.4$ & 0.652 & 0.648 & 1.595 & 1.557 & 1.493 & 1.484 & 2.879 & 2.758 & 1.207 & 1.125 \\
\hline $0.4-0.491$ & 0.669 & 0.664 & 1.643 & 1.640 & 1.538 & 1.583 & 2.935 & 2.961 & 1.412 & 1.248 \\
\hline
\end{tabular}

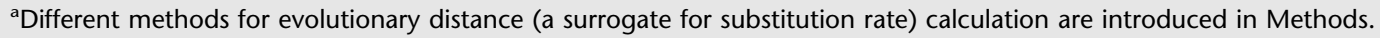

and sequence evolution rate. This is compatible with the previous reports on the connection between interactivity and dispensability (Jeong et al. 2001) and with the general notion that scalefree networks, such as the network of protein-protein interactions, are tolerant to error (random elimination of weakly connected nodes) but are highly vulnerable to attack (directed elimination of the hub; Albert et al. 2000; Barabasi 2002). Because protein-protein interaction domains generally show limited sequence conservation (whereas structure conservation is crucial), it is perhaps not unexpected that the connection between interactivity and sequence evolution rate could be detected (at best) only as a relatively weak statistical trend. Surprisingly, however, the observations reported here indicate that gene expression level more strongly correlated with sequence evolution rate than with PGL. Generally, one would expect the same trends to be seen with dispensability, interactivity, and expression level. If validated by further analysis of more robust and extensive expression data, this inversion could suggest a nontrivial connection between expression level and sequence conservation, the nature of which remains to be explored.

PGL and sequence evolution rate are measures of evolutionary conservation that seem to capture substantially different aspects of evolution. PGL is a much more direct reflection of the biological dispensability of a gene, whereas sequence evolution rate depends largely on the selective constraints on protein structure and sequence; the extent of these constraints depends on the nature of the protein function. We showed here that PGL and sequence evolution rate are moderately dependent; that is, highly constrained proteins are lost during evolution significantly less often than are weakly constrained ones. With the small set of seven eukaryotic genomes analyzed here, PGL is a coarse measure, and a much more refined analysis will become feasible as the collection of sequenced eukaryotic genomes grows (with prokaryotic genomes, of which a large database is already available, this type of analysis is hampered by widespread HGT, which could be hard to distinguish from gene loss; Snel et al. 2002; Kunin and Ouzounis 2003; Mirkin et al. 2003). Combined with improved data on gene dispensability, expression, and protein interactivity, such studies should take us closer to an understanding of the prevailing trends in genome evolution.

\section{METHODS}

\section{The KOG Data}

The KOGs were constructed largely as described previously (Tatusov et al. 1997, 2001), with minor modifications (Tatusov et al. 2003), and are available at http://www.ncbi.nlm.nih.gov/COG/ new/shokog.cgi and via ftp at ftp://ftp.ncbi.nih.gov/pub/COG/ $\mathrm{KOG} /$. If a KOG included more than one protein from one or more species (paralogs), the most conserved ortholog from the respective species was chosen. The sequences from the respective
KOG were compared to each other by using the BLASTP program (Altschul et al. 1997), and for each species, the sequence that had the best cumulative score with the sequences from the other species was selected.

\section{Other Data}

The data on gene knockout effects in yeast were primarily from Giaever et al. (2002). The SGD database (http://genome-www. stanford.edu/Saccharomyces/) was used to collect the knockout viability data for each individual gene. For KOGs with multiple yeast paralogs, only the most conserved paralog identified as described above was considered.

The GRID database was used as the source of data on protein-protein interactions (Breitkreutz et al. 2003). All duplicate interactions were collapsed into one entry. Absence of interactions in GRID for a given gene was interpreted as zero interactions.

Average expression levels of C. elegans genes were from Hill et al. (2000). Expression levels of human genes were estimated in the following fashion: The human CDSs were used as queries in a BLASTN search against the dbEST database. Hits with $>98 \%$ identity for alignment length $>400$ nt or with $>95 \%$ identity for alignment between 100 to $400 \mathrm{nt}$ were tallied, and the number of ESTs was taken as the expression level for the respective gene. Expression levels of yeast genes were obtained from the published microarray analysis by averaging the control (no diauxic shift) data (DeRisi et al. 1997). For all three organisms, gene expression data were mapped to KOGs, and if more than one paralog was present in a KOG, the maximum expression level for the given organism was assigned to the KOG.

\section{Divergence Times of E. cuniculi, S. cerevisiae, and S. pombe}

Phylogenetic trees for CDC28 kinase, glyceraldehyde-3-phosphate dehydrogenase (GPDH), small chain of ribonucleosidediphosphate reductase (RDR), and triosephosphate isomerase (TIM) families were constructed by using the Mega and ProtML packages (Adachi and Hasegawa 1992; Kumar et al. 1994). The lengths of the branches connecting E. cuniculi, S. cerevisiae, and $S$. pombe were taken to be proportional to the divergence times for these lineages. The divergence times were calculated by using the estimates for the other eukaryotic lineages (Wang et al. 1999). The ratio of the previously estimated times since divergence to branch lengths for $A$. thaliana, $H$. sapiens, C. elegans, and D. melanogaster was used to calibrate the branches of the tree in years. An average estimate over the CDC28, GPDH, RDR, and TIM families was used as the estimate of the time of divergence of $E$. cuniculi, S. cerevisiae, and S. pombe.

\section{PGL Calculations}

By using the published estimates (Wang et al. 1999) and our own estimates for the divergence times of E. cuniculi, S. cerevisiae, and $S$. pombe, specific divergence times were assigned to each internal node (ancestral form) in the phylogenetic tree of the eukaryotic crown group (Fig. 1A). Given a phyletic distribution pattern, 
branches of the tree associated with gene loss $\left(B_{L}\right)$ can be identified (Fig. 1B,C). Designating those branches of the tree, in which the given gene was preserved $B_{P}$, we have

$$
\mathrm{PGL}=\Sigma B_{L} /\left(\Sigma B_{P}+\Sigma B_{L}\right)
$$

In terms of Fig. 1, B and $\mathrm{C}$, this is the ratio of the sum of the lengths of blue branches to the sum of the lengths of all colored branches. Thus, for a gene present in Arabidopsis, human, and C. elegans but lost in the Drosophila branch and the FungiMicrosporidia branch (Fig. 1B),

$$
\begin{aligned}
\mathrm{PGL}= & (1063+0) /(1642+100+322+1220+157+1063 \\
& +1063+0)=0.19
\end{aligned}
$$

Similarly, for a gene found in Arabidopsis and the two yeast species (lost in the Metazoa branch and in the E. cuniculi branch, Fig. 1C),

$$
\begin{aligned}
\mathrm{PGL}= & (322+1542) /(1642+100+705+837+837+322 \\
& +1542)=0.31
\end{aligned}
$$

\section{Calculation of Evolutionary Distance Between Protein Sequences}

Evolutionary distances between proteins in a KOG were calculated from multiple alignments. To obtain the $P$-distance multiple alignments of protein sequences were constructed, and distances between orthologs were calculated as the proportion of different amino acids. All positions in the alignment containing a deletion or insertion in at least one of the sequences were removed prior to calculating $P$-distance. $P$-distances were measured relative to $A$. thaliana orthologs for all KOGs; their mean value was used as the distance characteristic for the given KOG. Similarly, evolutionary distances between proteins was calculated by using the PAM (Dayhoff et al. 1983) or JTT (Jones et al. 1992) substitution matrices and the mean distance from $A$. thaliana to other species was used for further analysis. The three kingdom mean distance was calculated as the unweighted average of the mean distances among plants, animals, and fungi. JTT matrix distances were also calculated with $\gamma$-correction by using the Protdist program with the $\alpha$-parameter of 1.0 (Felsenstein 1996).

\section{ACKNOWLEDGMENTS}

We thank Fyodor Kondrashov for help in the expression data analysis and I. King Jordan and Roland Walker for useful discussions.

The publication costs of this article were defrayed in part by payment of page charges. This article must therefore be hereby marked "advertisement" in accordance with 18 USC section 1734 solely to indicate this fact.

\section{REFERENCES}

Adachi, J. and Hasegawa, M. 1992. MOLPHY: Programs for molecular phylogenetics. Institute of Statistical Mathematics, Tokyo, Japan.

Albert, R., Jeong, H., and Barabasi, A.L. 2000. Error and attack tolerance of complex networks. Nature 406: 378-382.

Altschul, S.F., Madden, T.L., Schaffer, A.A., Zhang, J., Zhang, Z., Miller, W., and Lipman, D.J. 1997. Gapped BLAST and PSI-BLAST: A new generation of protein database search programs. Nucleic Acids Res. 25: 3389-3402.

Aravind, L., Watanabe, H., Lipman, D.J., and Koonin, E.V. 2000. Lineage-specific loss and divergence of functionally linked genes in eukaryotes. Proc. Natl. Acad. Sci. 97: 11319-11324.

Barabasi, A.L. 2002. Linked: The new science of networks. Perseus Press, New York.

Baumann, P., Baumann, L., Lai, C.Y., Rouhbakhsh, D., Moran, N.A., and Clark, M.A. 1995. Genetics, physiology, and evolutionary relationships of the genus Buchnera: Intracellular symbionts of aphids. Annu. Rev. Microbiol. 49: 55-94.

Breitkreutz, B.J., Stark, C., and Tyers, M. 2003. The GRID: The General Repository for Interaction Datasets. Genome Biol. 4: R23.

Bromham, L. and Penn, D. 2003. The modern molecular clock. Nat. Rev. Genet. 4: 216-224.

Dayhoff, M.O., Barker, W.C., and Hunt, L.T. 1983. Establishing homologies in protein sequences. Methods Enzymol. 91: 524-545.
DeRisi, J.L., Iyer, V.R., and Brown, P.O. 1997. Exploring the metabolic and genetic control of gene expression on a genomic scale. Science 278: $680-686$.

Farris, J.S. 1977. Phylogenetic analysis under Dollo's law. Syst. Zool. 26: $77-88$.

Felsenstein, J. 1996. Inferring phylogenies from protein sequences by parsimony, distance, and likelihood methods. Methods Enzymol. 266: $418-427$.

Fraser, H.B., Hirsh, A.E., Steinmetz, L.M., Scharfe, C., and Feldman, M.W. 2002. Evolutionary rate in the protein interaction network. Science 296: 750-752.

Giaever, G., Chu, A.M., Ni, L., Connelly, C., Riles, L., Veronneau, S., Dow, S., Lucau-Danila, A., Anderson, K., Andre, B., et al. 2002. Functional profiling of the Saccharomyces cerevisiae genome. Nature 418: $387-391$

Grishin, N.V., Wolf, Y.I., and Koonin, E.V. 2000. From complete genomes to measures of substitution rate variability within and between proteins. Genome Res. 10: 991-1000.

Hedges, S.B. 2002. The origin and evolution of model organisms. Nat. Rev. Genet. 3: 838-849.

Hedges, S.B. and Kumar, S. 2003. Genomic clocks and evolutionary timescales. Trends Genet. 19: 200-206.

Hedges, S.B., Chen, H., Kumar, S., Wang, D.Y., Thompson, A.S., and Watanabe, H. 2001. A genomic timescale for the origin of eukaryotes. BMC Evol. Biol. 1: 4.

Hill, A.A., Hunter, C.P., Tsung, B.T., Tucker-Kellogg, G., and Brown, E.L. 2000. Genomic analysis of gene expression in C. elegans. Science 290: $809-812$.

Hirsh, A.E. and Fraser, H.B. 2001. Protein dispensability and rate of evolution. Nature 411: 1046-1049.

Hurst, L.D. and Smith, N.G. 1999. Do essential genes evolve slowly? Curr. Biol. 9: 747-750.

Jeong, H., Mason, S.P., Barabasi, A.L., and Oltvai, Z.N. 2001. Lethality and centrality in protein networks. Nature 411: $41-42$.

Jones, D.T., Taylor, W.R., and Thornton, J.M. 1992. The rapid generation of mutation data matrices from protein sequences. Comput. Appl. Biosci. 8: 275-282.

Jordan, I.K., Rogozin, I.B., Wolf, Y.I., and Koonin, E.V. 2002. Essential genes are more evolutionarily conserved than are nonessential genes in bacteria. Genome Res. 12: 962-968.

Jordan, I.K., Wolf, Y.I., and Koonin, E.V. 2003. No simple dependence between protein evolution rate and the number of protein-protein interactions: Only the most prolific interactors tend to evolve slowly. BMC Evol. Biol. 3: 1.

Kamath, R.S., Fraser, A.G., Dong, Y., Poulin, G., Durbin, R., Gotta, M., Kanapin, A., Le Bot, N., Moreno, S., Sohrmann, M., et al. 2003. Systematic functional analysis of the Caenorhabditis elegans genome using RNAi. Nature 421: 231-237.

Katinka, M.D., Duprat, S., Cornillot, E., Metenier, G., Thomarat, F., Prensier, G., Barbe, V., Peyretaillade, E., Brottier, P., Wincker, P., et al. 2001. Genome sequence and gene compaction of the eukaryote parasite Encephalitozoon cuniculi. Nature 414: 450-453.

Kimura, M. 1983. The neutral theory of molecular evolution. Cambridge University Press, Cambridge.

Koonin, E.V. and Mushegian, A.R. 1996. Complete genome sequences of cellular life forms: Glimpses of theoretical evolutionary genomics. Curr. Opin. Genet. Dev. 6: 757-762.

Kumar, S., Tamura, K., and Nei, M. 1994. MEGA: Molecular Evolutionary Genetics Analysis software for microcomputers. Comput. Appl. Biosci. 10: 189-191.

Kunin, V. and Ouzounis, C.A. 2003. The balance of driving forces during genome evolution in prokaryotes. Genome Res. 13: $1589-1594$

Lipman, D.J., Souvorov, A., Koonin, E.V., Panchenko, A.R., and Tatusova, T.A. 2002. The relationship of protein conservation and sequence length. BMC Evol. Biol. 2: 20.

Mirkin, B.G., Fenner, T.I., Galperin, M.Y., and Koonin, E.V. 2003. Algorithms for computing parsimonious evolutionary scenarios for genome evolution, the last universal common ancestor and dominance of horizontal gene transfer in the evolution of prokaryotes. BMC Evol. Biol. 3: 2.

Moran, N.A. 2002. Microbial minimalism: Genome reduction in bacterial pathogens. Cell 108: 583-586.

Pal, C., Papp, B., and Hurst, L.D. 2001. Highly expressed genes in yeast evolve slowly. Genetics 158: 927-931.

. 2003. Genomic function: Rate of evolution and gene dispensability. Nature 421: 496-497.

Snel, B., Bork, P., and Huynen, M.A. 2002. Genomes in flux: The evolution of archaeal and proteobacterial gene content. Genome Res. 12: $17-25$.

Tatusov, R.L., Koonin, E.V., and Lipman, D.J. 1997. A genomic perspective on protein families. Science 278: 631-637.

\section{Genome Research}


Gene Loss and Its Correlates in Eukaryotic Evolution

Tatusov, R.L., Natale, D.A., Garkavtsev, I.V., Tatusova, T.A., Shankavaram, U.T., Rao, B.S., Kiryutin, B., Galperin, M.Y., Fedorova, N.D., and Koonin, E.V. 2001. The COG database: New developments in phylogenetic classification of proteins from complete genomes. Nucleic Acids Res. 29: 22-28.

Tatusov, R.L., Fedorova, N.D., Jackson, J.D., Jacobs, A.R., Kiryutin, B. Koonin, E.V., Krylov, D.M., Mazumder, R., Mekhedov, S.L., Nikolskaya, A.N., et al. 2003. The COG database: An updated version includes eukaryotes. BMC Bioinformatics (in press).

Wang, D.Y., Kumar, S., and Hedges, S.B. 1999. Divergence time estimates for the early history of animal phyla and the origin of plants, animals and fungi. Proc. R. Soc. Lond. B Biol. Sci. 266: $163-171$.

Wilson, A.C., Carlson, S.S., and White, T.J. 1977. Biochemical evolution. Annu. Rev. Biochem. 46: 573-639.
Wolfe, K.H. and Li, W.H. 2003. Molecular evolution meets the genomics revolution. Nat. Genet. 33(Suppl): 255-265.

\section{WEB SITE REFERENCES}

http://www.ncbi.nlm.nih.gov/COG/new/shokog.cgi; eukaryotic clusters of orthologous groups (KOGs).

http://genome-www.stanford.edu/Saccharomyces/; Saccharomyces Genome Database (SGD).

http://biodata.mshri.on.ca/grid/servlet/Index; the General Repository for Interaction Datasets (GRID).

Received April 27, 2003; accepted in revised form August 4, 2003. 




\section{Gene Loss, Protein Sequence Divergence, Gene Dispensability, Expression Level, and Interactivity Are Correlated in Eukaryotic Evolution}

Dmitri M. Krylov, Yuri I. Wolf, Igor B. Rogozin, et al.

Genome Res. 2003 13: 2229-2235

Access the most recent version at doi:10.1101/gr.1589103

References This article cites 38 articles, 10 of which can be accessed free at: http://genome.cshlp.org/content/13/10/2229.full.html\#ref-list-1

License

Email Alerting

Receive free email alerts when new articles cite this article - sign up in the box at the Service top right corner of the article or click here.

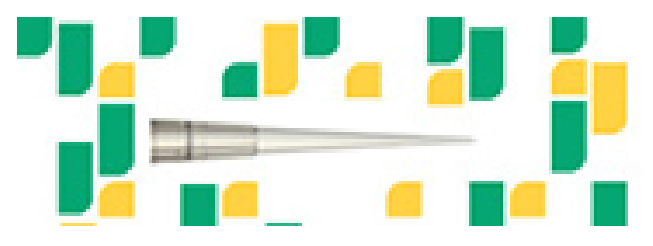

To subscribe to Genome Research go to: https://genome.cshlp.org/subscriptions 